\title{
Clinical Analysis of Macular Choroidal Thickness in Pseudoexfoliative Glaucoma and Primary Open-Angle Glaucoma
}

\author{
Fan Li $\mathbb{D},{ }^{1}$ Yiming Huo $\mathbb{D}^{\circ},{ }^{2}$ Lihua Ma $\mathbb{D},{ }^{1}$ Qing Zhang, ${ }^{1}$ Hengli Zhang $\mathbb{D},{ }^{1}$ Xiaowei Yan, \\ Yulei Geng $\mathbb{D}^{1}{ }^{1}$ and Guangxian Tang $\mathbb{D}^{1}$ \\ ${ }^{1}$ Department of Ophthalmology, Shijiazhuang People's Hospital, 365 Jianhua Road, Shijiazhuang, Hebei, China \\ ${ }^{2}$ Department of Ophthalmology, Shijiazhuang Aier Eye Hospital, 252 Zhonghua Road, Shijiazhuang, Hebei, China
}

Correspondence should be addressed to Lihua Ma; maria1001@126.com and Guangxian Tang; gxtykyy@126.com

Received 21 April 2021; Revised 27 October 2021; Accepted 29 October 2021; Published 16 November 2021

Academic Editor: Kofi Asiedu

Copyright (C) 2021 Fan Li et al. This is an open access article distributed under the Creative Commons Attribution License, which permits unrestricted use, distribution, and reproduction in any medium, provided the original work is properly cited.

Purpose. To evaluate the differences in macular choroidal thickness and volume among patients with pseudoexfoliative glaucoma (PXG), patients with primary open-angle glaucoma (POAG), and controls. Methods. A total of 50 PXG patients (50 eyes) and 56 POAG patients (56 eyes) were selected as the PXG group and the POAG group, respectively, in this case-control study. A total of 54 age-, gender-, IOP-, and axial length-matched healthy individuals (54 eyes) were selected as the control group. Enhanced-depth imaging-optical coherence tomography (EDI-OCT) was used to measure and analyze the choroidal thicknesses and volumes in 9 macular regions of all subjects. Results. The choroidal thicknesses in the central subfield (CSM), temporal inner macula (TIM), inferior inner macula (IIM), and temporal outer macula (TOM) and the mean macular choroidal thickness were significantly thinner in the PXG group than in the control group (all $P<0.05$ ). The choroidal volumes in the TIM, IIM, and TOM and the mean macular choroidal volume were significantly smaller in the PXG group than in the control group (all $P<0.05$ ). The choroidal thicknesses in the CSM and IIM and the mean macular choroidal thickness were significantly thinner in the PXG group than in the POAG group (all $P<0.05$ ). The choroidal volumes in the IIM and TOM and the mean macular choroidal volume were significantly smaller in the PXG group than in the POAG group (all $P<0.05$ ). Multivariable linear regression analysis showed that the mean macular choroidal thickness was significantly thinner in association with older subjects and longer axial length eyes. There was no association between the macular choroidal thickness of various macular regions and visual field mean defect (MD) in groups PXG and POAG (all $P>0.05$ ). Conclusions. The macular choroidal thicknesses and volumes (inferior and temporal) in PXG patients were thinner and smaller than those in POAG patients and healthy individuals. The role of choroidal thickness changes in the course of PXG remains unclear. A future prospective study is needed to better define these changes in PXG patients.

\section{Introduction}

Glaucoma is a serious eye disease that can lead to blindness. The number of glaucoma cases in Asia is expected to increase from 39 million in 2013 to 111.8 million in 2040 [1]. Pseudoexfoliative glaucoma (PXG) is a type of secondary open-angle glaucoma caused by pseudoexfoliative syndrome (PEX) that accounts for approximately $25 \%$ of all cases of open-angle glaucoma. It is well known that patients with PXG have higher intraocular pressure (IOP), greater diurnal variation in IOP, slower retrobulbar blood flow, more severe visual field damage, and more rapid progression than patients with primary open-angle glaucoma (POAG) [2-5].
High IOP is a major risk factor for open-angle glaucoma. Controlling IOP can effectively delay the progression of glaucoma. However, in some cases, loss of visual function is exacerbated even when IOP is under control, indicating that there might be other factors that affect disease progression [6]. Choroidal and systemic blood flow parameters may play a role in the development and progression of glaucoma [7]. The measurement of choroidal thickness can provide important information about the rate of the choroidal blood flow. Spectral domain-optical coherence tomography (SDOCT) can evaluate the choroid in vivo and offers high resolution and a fast scanning speed. The enhanced-depth imaging (EDI) mode can optimize OCT parameters, can 
TABLE 1: Baseline characteristics of the study groups are shown in three groups.

\begin{tabular}{|c|c|c|c|c|c|c|c|c|c|}
\hline Groups & $\begin{array}{l}\text { Eyes } \\
(n)\end{array}$ & $\begin{array}{c}\text { Gender } \\
(n) \\
\mathrm{M} / \mathrm{F}\end{array}$ & $\begin{array}{c}\text { Age } \\
\text { (years) }\end{array}$ & $\begin{array}{c}\mathrm{AL} \\
(\mathrm{mm})\end{array}$ & $\begin{array}{l}\mathrm{MD} \\
(\mathrm{dB})\end{array}$ & $\begin{array}{l}\text { Time } \\
\text { (days) }\end{array}$ & $\begin{array}{l}\text { Pretreatment } \\
\text { IOP }(\mathrm{mmHg})\end{array}$ & $\begin{array}{l}\text { Posttreatment } \\
\text { IOP }(\mathrm{mmHg})\end{array}$ & $\begin{array}{l}\text { Antiglaucoma } \\
\text { medications }(n)\end{array}$ \\
\hline PXG & 50 & $25 / 25$ & $75.36 \pm 6.75$ & $23.19 \pm 0.99$ & $-16.76 \pm 9.53$ & $58.70 \pm 50.56$ & $30.36 \pm 7.82$ & $16.30 \pm 2.97$ & $2.78 \pm 1.02$ \\
\hline POAG & 56 & $22 / 34$ & $73.66 \pm 6.23$ & $23.26 \pm 0.88$ & $-14.66 \pm 8.98$ & $65.75 \pm 49.92$ & $27.43 \pm 8.11$ & $16.16 \pm 2.37$ & $2.69 \pm 1.01$ \\
\hline Control & 54 & $20 / 34$ & $73.70 \pm 4.85$ & $23.22 \pm 0.89$ & $-1.04 \pm 0.41$ & - & - & $15.46 \pm 2.52$ & - \\
\hline$\chi^{2} / F$ & & 2.030 & 1.355 & 0.084 & 68.338 & 0.01 & 0.448 & 1.702 & 197.657 \\
\hline$P$ & & 0.362 & 0.261 & 0.919 & $<0.001$ & 0.473 & 0.062 & 0.186 & $<0.001$ \\
\hline
\end{tabular}

PXG: pseudoexfoliative glaucoma; POAG: primary open-angle glaucoma; M: male; F: female; IOP: intraocular pressure; AL: axial length; MD: mean defect; Time: duration between the first diagnosis and study enrolment.

image the full thickness of the choroid, and has high repeatability and reproducibility [8]. Previous studies on macular choroidal thickness in PXG and POAG have obtained mixed conclusions. One study has identified that patients affected by advanced POAG damage have a thinner choroidal thickness compared with normal subjects [6]. However, another study suggested that POAG was not significantly associated with a marked thinning or thickening of the choroid based on EDI-OCT measurements [9]. Dursun et al. [10] reported that macular and peripapillary choroidal thicknesses were decreased in PXG. In the present study, the macular choroidal thickness and volume in the eyes of Chinese patients with PXG and POAG were measured using EDI-OCT to investigate the changes in macular choroidal thickness in PEX and POAG eyes and to analyze the role of the choroid in the progression of PXG.

\section{Materials and Methods}

2.1. Patients. A total of 106 patients treated in our hospital between October 2018 and October 2020 were recruited for this study. The 50 PXG patients (50 eyes) were included in the PXG group, and 56 POAG patients (56 eyes) were included in the POAG group. Another 54 sex-, age-, IOP-, and axial length-matched healthy volunteers (54 eyes) were included in the control group. Both groups of glaucoma patients were treated with antiglaucoma medications to reduce IOP. There were no significant differences in age, sex, axial length, or IOP between the three groups (Table 1).

PXG diagnostic criteria were that the characteristic features of ocular PEX could be observed under a slit-lamp microscope, such as the appearance of gray-white exfoliative material at the pupillary margin, iris surface, and anterior lens capsule, IOP $>21 \mathrm{mmHg}$, and glaucomatous optic nerve damage and visual field defects [11]. Diagnostic criteria for the normal control group were a normal-looking optic disc (no disc edge narrowing or optic disc hemorrhage), cup disc ratio $(C / D) \leq 0.3$, binocular difference $\leq 0.2$, IOP $\leq 21 \mathrm{mmHg}$, and normal examination of the visual field and chamber angle.

The inclusion criteria were as follows: (1) It meets the diagnostic criteria of PXG and POAG. (2) The age of the subjects was $\geq 60$ years. (3) The equivalent spherical degree was $\leq \pm 6.0 \mathrm{D}$, and the cylindrical degree was $\leq \pm 3.0 \mathrm{D}$.

The exclusion criteria were other types of glaucoma (such as closed-angle glaucoma and secondary glaucoma); other ophthalmic diseases, such as corneal opacity, lens opacity, or other ocular diseases affecting the examination; previous history of ocular surgery or ocular trauma; retinal or macular diseases of the fundus; and systemic diseases such as hypertension and diabetes. This study followed the Helsinki Declaration and was approved by the ethics committee of Shijiazhuang People's Hospital (No.2018008). All subjects and their guardians signed informed consent forms.

2.2. Routine Examinations. All subjects underwent comprehensive eye examinations, including vision tests, slitlamp microscopy, IOP measurement (Goldmann applanation tonometer), axial length measurement, gonioscopy, and fundus and visual field examinations.

2.3. OCT Procedure. All subjects underwent the SD-OCT (Spectralis HRA + OCT, Heidelberg, Germany). The measurement illustration of macular choroidal thickness is shown in Figure 1. The macular thickness and volume were scanned using the EDI mode of the SD-OCT macular thickness map examination procedure. For specific measurement methods, refer to previous studies [12]. On each scanned image, the inscribed segmentation line was labeled on the retinal pigment epithelium/Bruch membrane interface, and the outer segmentation line was placed on the scleral/choroidal interface to represent the internal and external choroidal boundaries, shown in Figure 2. The choroidal thickness measurements were performed by the same technician.

2.4. Visual Field Procedure. The visual fields of all subjects were examined using the SITA-Fast 30-2 examination procedure and a Humphrey-750i visual field analyzer (Carl Zeiss, Germany). The reliability criteria included a fixation loss rate of $<20 \%$, a false negative rate of $<15 \%$, and a false positive rate of $<15 \%$. Individuals who did not meet the criteria were excluded.

2.5. Statistical Analyses. Data were performed using SPSS 21.0 statistical software. The mean and standard deviations $(\mathrm{M} \pm \mathrm{SD})$ of the above parameters were calculated. One-way ANOVA was performed for comparisons of age, axial length, IOP, visual field mean defect (MD), choroidal thickness, and volume among the three groups. An LSD $t$ test was used for pairwise comparisons. Multivariance linear 


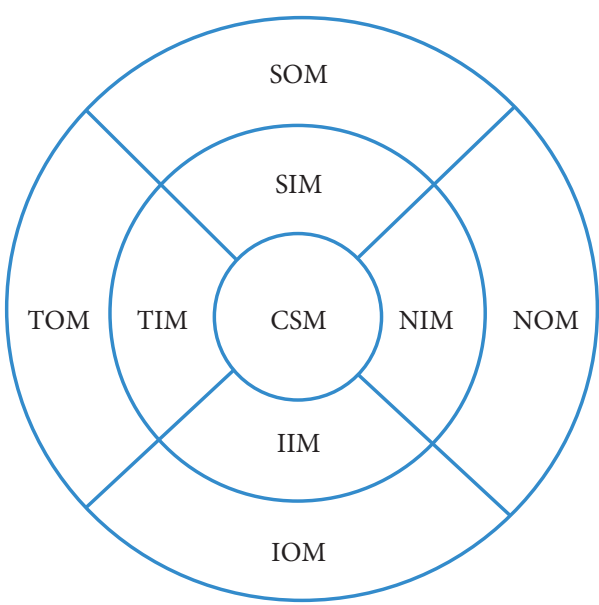

FIGURE 1: Measurement illustration of macular choroidal thickness at nine locations (reproduced from Li et al. [12]). CSM: central subfield macula; NIM: nasal inner macula; SIM: superior inner macula; IIM: inferior inner macula; TIM: temporal inner macula; NOM: nasal outer macula; SOM: superior outer macula; IOM: inferior outer macula; TOM: temporal outer macula.

regression analysis was done with stepwise modeling for determining the factors which influence mean macular choroidal thicknesses. Pearson's correlation analysis was used to analyze the correlation between macular thickness and visual field MD in PXG and POAG. Differences with $P<0.05$ were considered statistically significant.

\section{Results}

The mean macular choroidal thicknesses of the PXG, POAG, and control groups were $171.81 \pm 50.46 \mu \mathrm{m}$, $193.56 \pm 68.26 \mu \mathrm{m}$, and $197.74 \pm 46.82 \mu \mathrm{m}$, respectively, and the mean macular choroidal volumes were $0.52 \pm 0.15 \mu \mathrm{m}^{3}$, $0.59 \pm 0.21 \mu \mathrm{m}^{3}$, and $0.60 \pm 0.14 \mu \mathrm{m}^{3}$, respectively. The choroidal thicknesses in the central subfield (CSM), temporal inner macula (TIM), inferior inner macula (IIM), and temporal outer macula (TOM) and the mean macular choroidal thicknesses were significantly different among the three groups $(F=3.453,4.195,3.508,5.407$, and 3.149 , respectively, all $P<0.05)$. The choroidal thicknesses in the nasal inner macula (NIM), superior inner macula (SIM), nasal outer macula (NOM), superior outer macula (SOM), and inferior outer macula (IOM) were not significantly different among the three groups $(F=2.371,2.132,2.468$, 1.705 , and 2.828 , respectively, all $P>0.05$ ). The choroidal volumes in the TIM, IIM, and TOM and the mean macular choroidal volumes were significantly different among the three groups $(F=4.208,3.804,6.393$, and 3.264, respectively, all $P<0.05)$. The choroidal volumes in the CSM, NIM, SIM, NOM, SOM, and IOM were not significantly different $(F=2.340,2.459,2.141,2.508,1.765$, and 2.650, respectively, all $P>0.05)$.

Pairwise comparisons showed that the choroidal thicknesses in the CSM, TIM, IIM, and TOM and the mean macular choroidal thickness were significantly thinner in the PXG group than in the control group (all $P<0.05$ ) and that the choroidal volumes in the TIM, IIM, and TOM and the mean macular choroidal volumes were significantly smaller in the PXG group than in the control group (all $P<0.05$ ). The choroidal thicknesses in the CSM and IIM and the mean macular choroidal thickness were significantly thinner in the PXG group than in the POAG group (all $P<0.05$ ), and the choroidal thicknesses in the TIM and TOM were not significantly different between the two groups (all $P>0.05$ ). The choroidal volumes in the IIM and TOM and the mean macular choroidal volume were significantly smaller in the PXG group than in the POAG group (all $P<0.05$ ), and the choroidal volumes in the TIM were not significantly different between the two groups $(P>0.05)$. The choroidal thicknesses in the CSM, TIM, IIM, and TOM and the mean macular choroidal thickness were not significantly different between the POAG group and the control group (all $P>0.05$ ), and the choroidal volumes in the TIM, IIM, and TOM and the mean macular choroidal volume were not significantly different between these two groups (all $P>0.05$ ) (Table 2).

Multivariance linear regression analysis was done with stepwise modeling in order to investigate the factors which influence mean macular choroidal thickness in PXG and POAG groups. The influence of age and $\mathrm{AL}$ on mean macular choroidal thickness was found to be statistically significant. The mean macular choroidal thickness was significantly thinner in association with older subjects and longer $\mathrm{AL}$ eyes $(P<0.01$, standardized regression coefficient $=-0.377 ; \quad P=0.01, \quad$ standardized regression coefficient $=-0.232$ ).

There was no association between CSM, NIM, SIM, TIM, IIM, NOM, SOM, TOM, IOM, mean choroidal thickness, and visual field defects in group PXG $(r=-0.081, P=0.575$; $r=-0.043, \quad P=0.765 ; \quad r=-0.141, \quad P=0.330 ; \quad r=-0.133$, $P=0.355 ; \quad r=-0.032, \quad P=0.826 ; \quad r=0.064, \quad P=0.660$; $r=-0.069, \quad P=0.635 ; \quad r=-0.170, \quad P=0.239 ; \quad r=-0.072$, $P=0.618 ; r=-0.082, P=0.571)$. There was no association between CSM, NIM, SIM, TIM, IIM, NOM, SOM, TOM, IOM choroidal thickness, and visual field defects in group POAG $(r=0.244, P=0.071 ; r=0.239, P=0.076 ; r=0.271$, $P=0.054 ; \quad r=0.237, \quad P=0.079 ; \quad r=0.158, \quad P=0.243$; $r=0.181, \quad P=0.182 ; \quad r=0.242, \quad P=0.072 ; \quad r=0.228$, $P=0.091 ; r=0.170, P=0.210 ; r=0.227, P=0.093)$.

\section{Discussion}

Glaucoma is a multifactorial process, and recent studies have speculated that choroid thickness is associated with glaucoma progression $[4,13]$. The present study adds to the growing work on the relationship between choroidal thickness and PXG or that between choroidal thickness and POAG. A study has shown that macular choroidal thickness in POAG patients is not significantly different from that in healthy individuals [14]. In contrast, Cennamo et al. [15] found that the macular choroidal thickness of POAG patients measured by SD-OCT was thicker than that of healthy individuals. Egrilmez et al. [16] found that the macular choroidal thickness of PEX patients was thinner than that of POAG patients and healthy individuals. Moghimi et al. [17] argued that the choroidal thicknesses and volumes in the central subfield subfoveal region, superior quadrant, and 

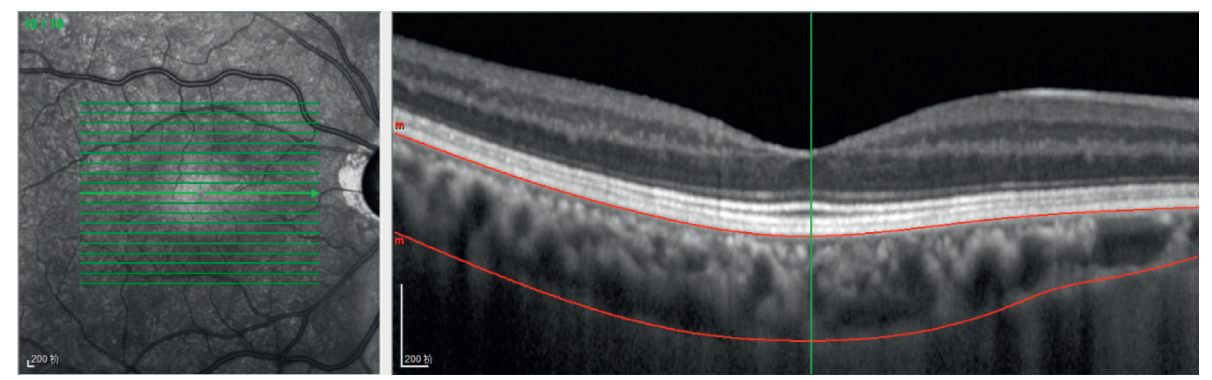

FIGURE 2: Optical coherence tomographic image (enhanced-depth imaging mode) for the measurement of the macular choroidal thickness.

TABLE 2: Comparisons of macular choroidal thickness by EDI-OCT in three groups.

\begin{tabular}{lccccc}
\hline \multirow{2}{*}{ Regions } & \multicolumn{2}{c}{ PXG (50 eyes) } & \multicolumn{2}{c}{ POAG (56 eyes) } & \multicolumn{2}{c}{ Control (54 eyes) } \\
& $\mathrm{TH}(\mu \mathrm{m})$ & $V\left(\mu \mathrm{m}^{3}\right)$ & $\mathrm{TH}(\mu \mathrm{m})$ & $V\left(\mu \mathrm{m}^{3}\right)$ & $\mathrm{TH}(\mu \mathrm{m})$ \\
\hline CSM & $181.46 \pm 56.46^{\mathrm{a}}$ & $0.14 \pm 0.05$ & $205.45 \pm 74.41^{\mathrm{b}}$ & $0.16 \pm 0.06$ & $212.13 \pm 55.34$ \\
NIM & $164.76 \pm 54.85$ & $0.26 \pm 0.09$ & $189.25 \pm 75.97$ & $0.30 \pm 0.12$ & $186.81 \pm 54.61$ \\
SIM & $192.10 \pm 59.54$ & $0.30 \pm 0.09$ & $207.59 \pm 68.53$ & $0.33 \pm 0.11$ & $216.35 \pm 51.66$ \\
TIM & $182.62 \pm 55.60^{\mathrm{a}}$ & $0.29 \pm 0.09^{\mathrm{a}}$ & $202.18 \pm 68.75$ & $0.32 \pm 0.11$ & $216.28 \pm 51.73$ \\
IIM & $169.64 \pm 58.91^{\mathrm{a}}$ & $0.27 \pm 0.09^{\mathrm{a}}$ & $203.07 \pm 81.16^{\mathrm{b}}$ & $0.32 \pm 0.13^{\mathrm{b}}$ & $0.34 \pm 0.04$ \\
NOM & $132.48 \pm 45.11$ & $0.70 \pm 0.24$ & $155.55 \pm 71.30$ & $0.83 \pm 0.38$ & $195.56 \pm 55.16$ \\
SOM & $191.32 \pm 54.86$ & $1.01 \pm 0.29$ & $201.89 \pm 67.57$ & $1.07 \pm 0.36$ & $211.93 \pm 45.34$ \\
TOM & $168.72 \pm 46.02^{\mathrm{a}}$ & $0.88 \pm 0.25^{\mathrm{a}}$ & $187.14 \pm 57.78$ & $0.99 \pm 0.31^{\mathrm{b}}$ & $201.30 \pm 46.34$ \\
IOM & $163.10 \pm 54.82$ & $0.86 \pm 0.29$ & $189.89 \pm 75.44$ & $1.00 \pm 0.41$ & $185.52 \pm 50.27$ \\
MM & $171.80 \pm 50.46^{\mathrm{a}}$ & $0.52 \pm 0.15^{\mathrm{a}}$ & $193.56 \pm 68.26^{\mathrm{b}}$ & $0.59 \pm 0.21^{\mathrm{b}}$ & 1.08 \\
\hline
\end{tabular}

PXG: pseudoexfoliative glaucoma; POAG: primary open-angle glaucoma; comparison between the PXG group and control group by LSD $t$-test, ${ }^{\mathrm{a}} P<0.05$; comparison between the PXG group and POAG group by LSD $t$-test, ${ }^{b} P<0.05$; CSM: central subfield macula; NIM: nasal inner macula; SIM: superior inner macula; IIM: inferior inner macula; TIM: temporal inner macula; NOM: nasal outer macula; SOM: superior outer macula; IOM: inferior outer macula; TOM: temporal outer macula; MM: mean macula; TH: thickness; $V$ : volume. Data are expressed as mean \pm standard deviation.

nasal quadrant of inner rings were all significantly lower in PEX patients than in healthy individuals, while there were no significant differences in the macular choroidal thickness after adjustment for age, gender, and axial length. Our previous studies $[12,18]$ found that the macular choroidal thicknesses of PXG eyes, fellow eyes without PXG, and PEX eyes were thinner than those of normal eyes; that the macular choroidal thicknesses of PXG eyes (except on the temporal region) were thinner than those of fellow eyes without PXG; and that macular choroidal thickness was not significantly different between PXG eyes and PEX eyes. Our previous studies also found that the choroidal thicknesses of PEX eyes with normal IOP were thinner than those of normal eyes and that the macular choroidal thickness became progressively thinner as PXG progressed. Therefore, we speculated that the macular choroidal thickness in PEX eyes changed before the IOP increased and that risk factors for PXG might include factors unrelated to IOP that might be related to hemodynamic changes caused by the impact of exfoliative material on the vascular structure [19]. To further elucidate the effect of exfoliative material and high IOP on choroidal thickness, this study performed a comparative analysis of the choroidal thicknesses of PXG and POAG eyes. After matching all three groups of subjects for age, gender, IOP, and axial length, we found that the macular choroidal thicknesses in PXG eyes were thinner than those in POAG and normal eyes, while there was no significant difference in macular choroidal thickness between POAG eyes and normal eyes. Koz et al. [20] detected glaucomatous optic nerve damage in PEX patients with normal IOP. They speculated that the presence of the highest IOP and greatest IOP fluctuation in PEX eyes may be important factors in the progression of glaucoma and that the presence of exfoliative material may be an independent risk factor for glaucomatous optic neuropathy. Exfoliative material has been proven to accumulate in small vascular endothelial cells and pericytes and to regulate microcirculation, and the deposition of exfoliative material in blood vessels can cause insufficient circulation or occlusion, leading to ischemic changes [8]. Kose and Tekeli [21] reported that the vascular densities around the optic disc and in the macula of PXG eyes were lower than those of the POAG eyes and considered the perfusion-related injury around the optic disc and in the macula as a risk factor for the progression of glaucoma, which is faster in PXG patients. The above studies all indicated that vascular factors may play an important role in the pathogenesis of PXG.

This study still has some limitations. First, the number of patients enrolled in the study was relatively small. This study set stringent inclusion criteria, such as age and axial length, to ensure a strict match between the experimental groups and the control group. Second, due to the lack of automatic measurement software, manual delineation of the choroidal margin might have introduced some measurement errors. Third, previous studies have shown that diurnal changes in choroidal thickness occur [22]. However, OCT examinations of different participants were performed at random hours, which introduced some errors. Fourth, patients with a 
history of hypertension were not included in this study, and the influences of systolic blood pressure, diastolic blood pressure, and ocular perfusion pressure on choroidal thickness were not evaluated.

\section{Conclusions}

The macular choroidal thicknesses and volumes (inferior and temporal) in PXG patients were thinner and smaller than those in POAG patients and healthy individuals. The role of choroidal thickness changes in the course of PXG remains unclear. The impact of macular choroidal thickness on glaucoma needs to be further investigated in extensive multicenter trials.

\section{Data Availability}

The research data used to support the findings of this study are available from the corresponding author upon request.

\section{Conflicts of Interest}

All authors declare that there are no conflicts of interest.

\section{Acknowledgments}

This research project was supported by the Funds for the Key Project Guidance Program of Medical Science in Hebei Province of China (20190164).

\section{References}

[1] Y.-C. Tham, X. Li, and T. Y. Wong, "Global prevalence of glaucoma and projections of glaucoma burden through 2040: a systematic review and meta-analysis," Ophthalmology, vol. 121, pp. 2081-2090, 2014.

[2] C. O. Mehmet, Z. Aktas, B. K. Yildiz, M. Hasanreisoglu, and B. Hasanreisoglu, "Retinal vessel diameters and their correlation with retinal nerve fiber layer thickness in patients with pseudoexfoliation syndrome," International Journal of Ophthalmology, vol. 8, no. 2, pp. 332-336, 2015.

[3] P. Plateroti, A. M. Plateroti, S. Abdolrahimzadeh, and G. Scuderi, "Pseudoexfoliation syndrome and pseudoexfoliation glaucoma: a review of the literature with updates on surgical management," Journal of Ophthalmology, vol. 2015, Article ID 370371, 9 pages, 2015.

[4] G. Cristini, G. Cennamo, and P. Daponte, "Choroidal thickness in primary glaucoma," Ophthalmologica, vol. 202, no. 2, pp. 81-85, 1991.

[5] E. Vesti and T. Kivelä, "Exfoliation syndrome and exfoliation glaucoma," Progress in Retinal and Eye Research, vol. 19, no. 3, pp. 345-368, 2000.

[6] R. Sacconi, N. Deotto, T. Merz, R. Morbio, S. Casati, and G. Marchini, "SD-OCT choroidal thickness in advanced primary open-angle glaucoma," Journal of Glaucoma, vol. 26, no. 6, pp. 523-527, 2017.

[7] L. Abegão Pinto, K. Willekens, K. Van Keer, A. Shibesh, G. Molenberghs, and E. Vandewalle, "Ocular blood flow in glaucoma - the leuven eye study," Acta Ophthalmologica, vol. 94, no. 6, pp. 592-598, 2016.

[8] Z. Lin, S. Huang, P. Huang, L. Guo, X. Shen, and Y. Zhong, "The diagnostic use of choroidal thickness analysis and its correlation with visual field indices in glaucoma using spectral domain optical coherence tomography," PLoS One, vol. 12, no. 12, p. e0189376, 2017.

[9] W. Wang and X. Zhang, "Choroidal thickness and primary open-angle glaucoma: a cross-sectional study and metaanalysis," Investigative Opthalmology \& Visual Science, vol. 55, no. 9, pp. 6007-6014, 2014.

[10] A. Dursun, A. V. Ozec, O. Dogan et al., "Evaluation of choroidal thickness in patients with pseudoexfoliation syndrome and pseudoexfoliation glaucoma," Journal of Ophthalmology, vol. 2016, Article ID 3545180, 5 pages, 2016.

[11] N. Yüksel, V. L. Karabaş, A. Arslan, A. Demirci, and Y. Çağlar, "Ocular hemodynamics in pseudoexfoliation syndrome and pseudoexfoliation glaucoma," Ophthalmology, vol. 108, no. 6, pp. 1043-1049, 2001.

[12] F. Li, Q. Shang, G. Tang et al., "Analysis of peripapillary and macular choroidal thickness in eyes with pseudoexfoliative glaucoma and fellow eyes," Journal of Ophthalmology, vol. 2020, Article ID 9634543, 7 pages, 2020.

[13] C. G. Vasconcelos De Moraes, A. S. Castro Reis, A. F. de Sá Cavalcante, M. E. Sano, and R. Susanna, "Choroidal expansion during the water drinking test," Graefe's Archive for Clinical and Experimental Ophthalmology, vol. 247, no. 3, pp. 385-389, 2009.

[14] J. B. Jonas, P. Steinmetz, T. M. Forster, F. C. Schlichtenbrede, and B. C. Harder, "Choroidal thickness in open-angle glaucoma," Journal of Glaucoma, vol. 24, no. 8, pp. 619-623, 2015.

[15] G. Cennamo, M. Finelli, G. Iaccarino, G. de Crecchio, and G. Cennamo, "Choroidal thickness in open-angle glaucoma measured by spectral-domain scanning laser ophthalmoscopy/optical coherence tomography," Ophthalmologica, vol. 228, no. 1, pp. 47-52, 2012.

[16] E. D. Egrilmez, S. K. Ugurlu, S. S. Atik, and Y. Z. Guven, "The effect of pseudoexfoliation syndrome on choroidal thickness in open-angle glaucoma," Arquivos Brasileiros de Oftalmologia, vol. 82, no. 5, pp. 400-406, 2019.

[17] S. Moghimi, M. Mazloumi, M. K. Johari et al., "Comparison of macular choroidal thickness in patients with pseudoexfoliation syndrome to normal control subjects with enhanced depth SD-OCT imaging," Journal of Current Ophthalmology, vol. 29, no. 4, pp. 258-263, 2017.

[18] F. Li, L. Ma, Y. Geng et al., "Comparison of macular choroidal thickness and volume between pseudoexfoliative glaucoma and pseudoexfoliative syndrome," Journal of Ophthalmology, vol. 2020, Article ID 8886398, 5 pages, 2020.

[19] E. T. Detorakis, A. K. Achtaropoulos, E. E. Drakonaki, and V. P. Kozobolis, "Hemodynamic evaluation of the posterior ciliary circulation in exfoliation syndrome and exfoliation glaucoma," Graefe's Archive for Clinical and Experimental Ophthalmology, vol. 245, no. 4, pp. 516-521, 2007.

[20] O. G. Koz, M. F. Turkcu, A. Yarangumeli, C. Koz, and G. Kural, "Normotensive glaucoma and risk factors in normotensive eyes with pseudoexfoliation syndrome," Journal of Glaucoma, vol. 18, no. 9, pp. 684-688, 2009.

[21] H. C. Kose and O. Tekeli, "Optical coherence tomography angiography of the peripapillary region and macula in normal, primary open angle glaucoma, pseudoexfoliation glaucoma and ocular hypertension eyes," International Journal of Ophthalmology, vol. 13, no. 5, pp. 744-754, 2020.

[22] T. Kinoshita, Y. Mitamura, K. Shinomiya, M. Egawa, A. Iwata, and A. Fujihara, "Diurnal variations in luminal and stromal areas of choroid in normal eyes," British Journal of Ophthalmology, vol. 101, 2016. 